\title{
Depression and Distress Predict Time to Cardiovascular Disease in Dementia Caregivers
}

\author{
Brent T. Mausbach \\ University of California at San Diego and Veterans Affairs \\ San Diego Health Care System \\ Yaron G. Rabinowitz \\ Walter Reed Army Medical Center
}

\author{
Thomas L. Patterson \\ University of California at San Diego and Veterans Affairs \\ San Diego Health Care System \\ Igor Grant \\ University of California at San Diego and Veterans Affairs \\ San Diego Health Care System
}

\author{
Richard Schulz \\ University of Pittsburgh
}

\begin{abstract}
Objective: This study assessed the impact of depressive symptoms and distress from patient problem behaviors on time to developing a diagnosis of cardiovascular disease (CVD) in a sample of 643 dementia family caregivers. Design: A longitudinal, prospective design was used. Over an 18-month period, caregivers free from a CVD diagnosis at baseline were assessed at 6,12, and 18-month follow-ups for the onset of CVD. Main Outcome Measures: Days to the onset of CVD was the primary outcome. Results: Over the length of the study, 32 participants (5\%) reported a diagnosis of CVD. After adjusting for sociodemographic and health factors (e.g., high blood pressure, age, smoking history), greater depressive symptoms ( $p=.040)$ and distress from patient problem behaviors $(p=.034)$ were significant predictors of time to CVD diagnosis. Conclusion: This study suggests that increased depressive symptoms and reaction to patient problem behaviors (i.e., distress) may increase caregivers' risk for experiencing negative health outcomes, specifically CVD.
\end{abstract}

Keywords: cardiovascular disease, dementia caregiving, depression, distress

It has been suggested that the high levels of stress associated with caring for a loved one with dementia provide an excellent model for examining the relations between stress and physical health (Grant, 1999). To be sure, an abundance of research indicates that caregiving is associated with increased risk for a number of physical health consequences (Vitaliano, Zhang, \& Scanlan, 2003), such as hyperlipidemia (Vitaliano, Russo, \& Niaura, 1995), hyperglycemia and hyperinsulinemia (Vitaliano, Scanlan, Krenz, \& Fujimoto, 1996), slowing of wound healing (Kiecolt-Glaser, Marucha, Malarkey, Mercado, \& Glaser, 1995), and impaired immune functioning (Kiecolt-Glaser, Glaser, Gravenstein,

Brent T. Mausbach, Thomas L. Patterson, and Igor Grant, Department of Psychiatry, University of California at San Diego, Veterans Affairs San Diego Health Care System; Yaron G. Rabinowitz, Walter Reed Army Medical Center; Richard Schulz, Department of Psychiatry and University Center for Social and Urban Research, University of Pittsburgh.

This research was supported by the National Institute on Aging and the National Institute of Nursing Research (under awards AG23989, AG15301, AG13313, AG13297, AG13289, AG13265, AG13255, AG13305, and NR13269).

Correspondence concerning this article should be addressed to Brent T. Mausbach, Department of Psychiatry (0680), University of California, San Diego, 9500 Gilman Drive, La Jolla, CA 92093-0680. E-mail: bmausbach@ucsd.edu
Malarkey, \& Sheridan, 1996; Mills et al., 2004; Mills, Yu, Ziegler, Patterson, \& Grant, 1999; Mills et al., 1997). Perhaps the most notable example of the physical health consequences of caregiving was the work by Schulz and Beach (1999), who found that caregivers reporting significant strain were $63 \%$ more likely to die over a 4-year period than a comparable sample of noncaregivers.

More recently, it has been noted that caregivers are at increased risk for developing hypertension (Shaw et al., 1999) and cardiovascular disease (Lee, Colditz, Berkman, \& Kawachi, 2003; Vitaliano et al., 2002). This increased risk is believed to be due, in part, to the development of a hypercoagulable state resulting from accelerated clotting or impaired fibrinolysis. Studies testing this model indicate that caregivers have exaggerated thrombinantithrombin III complexes (TAT) and fibrin D-dimer responses to stress (von Känel et al., 2001); factors which are known to contribute to thrombin formation (Pelzer, Schwarz, \& Heimburger, 1988) and fibrin formation and degradation (Lip \& Lowe, 1995), respectively. Other studies comparing caregivers with noncaregiving controls indicate that caregivers have elevated fibrin formation and overall hemostatic activation (von Kanel et al., 2005).

In order to promote caregiver health and to minimize caregiver mortality, it is important to determine specific caregiving factors which contribute to the onset of cardiovascular disease among caregivers. However, the specific aspects of caregiving that contribute to the onset of cardiovascular disease have not been clearly 
delineated in the extant literature. Lee and colleagues (2003) report that female caregivers who provide 9 or more hours of direct care per day are at increased risk of developing coronary artery disease. Other research, however, suggests that subjective distress (e.g., being upset by a loved-one's memory and behavior problems), may be related to cardiovascular risk factors, including heightened sympathetic arousal (Pike et al., 1997) which, over time, may cause an allostatic load and increase one's risk for developing cardiovascular disease. In the caregiver health effects study (Schulz \& Beach, 1999), mental or emotional strain was predictive of 4-year mortality among caregivers, although these effects were not specific to cardiovascular disease.

Depression, which is common among caregivers (Baumgarten et al., 1992; Cohen et al., 1990; Schulz, O'Brien, Bookwala, \& Fleissner, 1995; Williamson \& Schulz, 1993), is another factor which may contribute to the onset of cardiovascular disease (Anda et al., 1993; Ford et al., 1998; Lespérance, Frasure-Smith, Talajic, \& Bourassa, 2002; Musselman, Evans, \& Nemeroff, 1998), although no research to date has demonstrated this effect in a caregiving sample. However, studies of caregivers have found that depressive symptoms exacerbate the effects of psychological stress on catecholamine response (Mausbach et al., 2005) and D-dimer reactivity (von Känel et al., 2004). Therefore, in the context of chronic stress, depressive symptoms may produce molecular and vascular changes that increase caregivers' risk of developing cardiovascular disease.

Given the evidence that caregiver distress is associated with sympathetic arousal, hemostatic alterations, and caregiver mortality, and that depressive symptoms have been associated with risk for cardiovascular disease, it was hypothesized that depression and emotional distress resulting from care recipient problem behaviors would significantly predict time to onset of cardiovascular disease.

\section{Method}

\section{Study Population}

All participants were recruited into the national REACH (Resources for Enhancing Alzheimer's Caregiver Health) project, a multi-site research program sponsored by the National Institute on Aging and the National Institute on Nursing Research. The primary purpose of the REACH project was to test the effectiveness of multiple interventions for improving well-being in family caregivers of persons with Alzheimer's disease or related disorders (ADRD; Schulz et al., 2003; Wisniewski et al., 2003). The active treatment phase lasted approximately 6 months at each site.

The REACH project enrolled 1,222 caregivers between 1996 and 2000 from six sites in the United States: (a) Birmingham, AL, (b) Boston, MA, (c) Memphis, TN, (d) Miami, FL, (e) Palo Alto, $\mathrm{CA}$, and (f) Philadelphia, PA. In addition to Caucasian caregivers, a substantial number of African American and Hispanic caregivers were recruited. Each site obtained approval from their institutional review boards, and all participants provided written informed consent prior to enrollment.

\section{Evaluation}

At baseline and again at 6,12 , and 18 months postbaseline, a trained interviewer administered a standardized in-home assess- ment to collect a variety of physical health, medication, and psychosocial information.

Physical health and medication information. Participants were asked if they had been diagnosed by a physician with any of the following heart conditions: (a) heart disease, (b) heart attack, (c) congestive heart failure, (d) angina, or (e) myocardial infarction. If the participant reported a diagnosis of any of these conditions, they were classified as having cardiovascular disease. In addition, participants were asked if they currently smoked and if they had been diagnosed by a physician as having high blood pressure. Caregiver self-rated health was measured using four items that assessed perceived physical health (Ware, Kosinski, \& Keller, 1996). The first question asked respondents to rate their health on a scale ranging from "poor" to "excellent." The next three questions asked caregivers to compare their health to others (e.g., "I seem to get sick a little easier than other people"), with responses ranging from "definitely false" to "definitely true." The possible range of scores was 4-20, with higher scores indicating better health.

Prior to being interviewed, participants were instructed to gather all his/her medications into a bag. The interviewer recorded the names of all medications being taken by the caregiver. Medications were reviewed and assigned a drug code according to the Instant Drug Index (IDI) to illustrate their primary purpose(s) (e.g., anti-depressant, cardiovascular, pain, etc.). For the present study, participants' medications were coded (yes vs. no) as antidepressant or cardiovascular in nature.

Psychosocial functioning. The Center for Epidemiologic Studies-Depression scale (CES-D; Radloff, 1976) was used to measure the presence of depressive symptoms. Previous research indicates that a score of 16 or higher on this scale has been identified to adequately discriminate depressed and non-depressed individuals (Radloff \& Teri, 1986).

The Revised Memory and Behavior Problem Checklist (RMBPC; Teri et al., 1992) was used to measure caregiver reaction to care recipient problem behaviors. Specifically, caregivers were asked whether their care recipient manifested any 1 of 24 behavior problems (7 memory, 8 depressive, and 9 disruptive) during the past week. If caregivers responded "yes," they were asked how bothered or upset they were by each behavior using a 5-point Likert scale ranging from 0 (not at all) to 4 (extremely). For problem behaviors that were not endorsed, an upset score of 0 was assigned. A total reaction (i.e., distress) score was calculated by summing the upset scores, resulting in a range of scores from 0-96.

Sociodemographic variables. The following sociodemographic information was collected during the baseline assessment: (a) age, (b) sex, (c) ethnicity, and (d) education. Also, caregivers also reported years of caregiving and hours of care per day they provided to their care recipients.

\section{Analytic Methods}

Cox regression was used to examine the relationship between the two emotional distress measures (i.e., CES-D and RMBPC reaction scores) and time to onset of cardiovascular disease, while controlling for other predictors. "Survival" time was the number of days between the baseline interview and the last interview or diagnosis of cardiovascular disease. Table 1 provides information on all variables included in the analysis. Table 2 presents the results from the Cox regression model and both adjusted and 
Table 1

Participant Characteristics $(N=643)$

\begin{tabular}{lcr}
\hline \multicolumn{1}{c}{ Characteristics } & $M(S D)$ & $n(\%)$ \\
\hline Age (years) & $56.7(13.2)$ & \\
Sex & & $549(85)$ \\
$\quad$ Female & & $93(15)$ \\
$\quad$ Male & & $348(54)$ \\
Ethnicity & & $294(46)$ \\
$\quad$ Caucasian & & \\
$\quad$ Other & $13.00(2.6)$ & \\
Education (years) & $4.1(4.0)$ & \\
Years of caregiving & & $540(84)$ \\
Current smoker & & \\
$\quad$ No & & \\
$\quad$ Yes & & \\
High blood pressure & & \\
$\quad$ No & & \\
$\quad$ Yes & $14.8(3.5)$ & \\
Self-rated health & & \\
Taking antidepressant medication & & \\
$\quad$ No & & \\
$\quad$ Yes & & \\
Hours of care/day & & \\
CES-D score & & \\
RMBPC bother score & $11.6(6.9)$ \\
\hline
\end{tabular}

Note. $\mathrm{RMBPC}=$ Revised Memory and Behavior Problem Checklist; CES-D $=$ Center for Epidemiologic Studies Depression scale.

unadjusted relative risk (RR) ratios (from a Cox model with only that variable as a predictor). Emotional distress effects were tested by entering the RMBPC reaction and CES-D scores as linear variables. These variables were entered in a single step along with the following covariates: (a) age, (b) sex, (c) ethnicity, (d) years of education, (e) years of caregiving, (f) current smoking status (yes vs. no), (g) high blood pressure (yes vs. no), (h) self-rated health, (i) taking antidepressant medication(s) (yes vs. no), (j) whether or not the caregiver provided 9 or more hours of care per day, and $(\mathrm{k})$ whether or not the caregiver was assigned to an active REACH treatment condition.

\section{Results}

Of the 1,222 participants enrolled in the REACH project, 234 indicated during the baseline assessment they had already been diagnosed with heart disease. Because the focus of this study was to assess the onset of heart disease, these participants were not included in the analyses. In addition, 345 participants reported taking cardiovascular medication at the baseline assessment. Because cardiovascular medication may confound interpretation of results, it was considered as a covariate. However, none of these individuals developed heart disease during their 18-months of participation, thereby precluding it from being used as a covariate. As a result, these individuals were removed from the final analyses.

Table 1 reports descriptive statistics for all variables used in the analysis for the remaining 643 participants. The current sample of caregivers ranged in age from 22 to 91 years at baseline $(M=57$ years); $85 \%$ were women and $15 \%$ were men. Two-hundred-fifty (39\%) of the caregivers were classified as depressed. Statistical tests were conducted to compare sociodemographic and health characteristics of excluded and included participants. For these analyses, $t$ tests and chi-square analyses were used to compare continuous and dichotomous variables, respectively. As expected, participants reporting baseline cardiovascular disease or who were taking cardiovascular medications (i.e., excluded participants) were older $(t=11.24, p<.001)$, reported worse overall health $(t=10.75, p<.001)$, were more likely to be male $\left(\chi^{2}=14.08\right.$, $p<.001)$, a current smoker $\left(\chi^{2}=5.30, p<.05\right)$, and have high blood pressure $\left(\chi^{2}=525.25, p<.001\right)$.

After 18-months of follow-up, 32 participants (5\%) reported a diagnosis of cardiovascular disease. Of these, one (3.1\%) partici-

Table 2

Association of Sociodemographic, Health, and Caregiver Distress Factors With Onset of Heart Disease $^{a}$

\begin{tabular}{lcc}
\hline \multicolumn{1}{c}{ Variables } & $\begin{array}{c}\text { Unadjusted relative } \\
\text { risk (95\% CI) }\end{array}$ & $\begin{array}{c}\text { Adjusted relative } \\
\text { risk (95\% CI) }\end{array}$ \\
\hline $\begin{array}{l}\text { Sociodemographic factors } \\
\text { Age }\end{array}$ & $1.03(1.00-1.06)^{*}$ & $1.04(1.01-1.07)^{*}$ \\
Sex (Male) & $1.53(0.63-3.73)$ & $1.66(0.66-4.21)$ \\
Ethnicity (non-caucasian) & $0.80(0.40-1.63)$ & $0.89(0.40-1.99)$ \\
Education (years) & $0.98(0.86-1.11)$ & $1.02(0.87-1.19)$ \\
Years of caregiving & $1.04(0.97-1.12)$ & $1.03(0.95-1.12)$ \\
Current smoker & $1.31(0.54-3.19)$ & $1.54(0.59-4.06)$ \\
High blood pressure & $2.68(1.10-6.53)^{*}$ & $3.10(1.22-7.89)^{*}$ \\
Self-rated health & $1.04(0.94-1.16)$ & $1.11(0.98-1.26)$ \\
Taking antidepressant medication & $0.85(0.26-2.81)$ & $0.54(0.15-1.90)$ \\
$\quad$ Treatment (vs. control) & $1.04(0.50-2.16)$ & $1.13(0.52-2.43)$ \\
Caregiving distress factors & & $1.24(0.59-2.60)$ \\
>9 hours of caregiving/day & $1.51(0.74-3.11)$ & $1.03(1.02-1.05)^{*}$ \\
RMBPC bother score & $1.03(1.01-1.05)^{* *}$ & $1.04(1.00-1.08)^{*}$ \\
CES-D score & $1.03(1.00-1.06)$ & \\
\hline
\end{tabular}

Note. $\quad$ RMBPC $=$ Revised Memory and Behavior Problem Checklist CES-D $=$ Center for Epidemiologic Studies Depression scale; CI = confidence interval.

${ }^{\text {a }}$ Total number of participants is 643 . Total number of participants reporting an onset of heart disease is 32 (5\%).

${ }^{*} p<.05 .{ }^{* *} p<.01$. 
pant was diagnosed within the first 6 months of the study, 13 (40.6\%) were diagnosed between the 6 and 12-month follow-up, and $18(56.3 \%)$ were diagnosed between the 12 and 18-month follow-up. The mean time to onset was approximately 400 days (i.e., 13 months). Table 2 shows that after adjusting for sociodemographic and health factors, higher CES-D scores (RR, 1.04; 95\% CI, 1.00-1.08; $p=.040)$ and higher RMBPC reaction scores (RR, $1.03 ; 95 \%$ CI, 1.00-1.05; $p=.034$ ) were significantly related to time to CVD onset. Other significant predictors included age (RR, 1.04; 95\% CI, 1.01-1.07; $p=.024)$ and a diagnosis of high blood pressure (RR, 3.10; 95\% CI, 1.22-7.89; $p=.018$ ).

\section{Discussion}

After controlling for other known risk factors of cardiovascular disease, such as high blood pressure, smoking status, and sociodemographic characteristics (e.g., age), we found that increased depressive symptoms and reaction to $\mathrm{CR}$ problem behaviors were independent significant predictors of time to developing cardiovascular disease within 18 months. These results support previous research indicating that emotional strain is predictive of the development of heart disease (Anda et al., 1993; Ford et al., 1998; Lespérance et al., 2002; Musselman et al., 1998) and ultimately of mortality (Schulz \& Beach, 1999). Additionally, this is the first study to extend these findings to a sample of dementia family caregivers. Perhaps most importantly, these results, combined with evidence that emotional distress in caregivers promotes both SAM responsivity to psychological stress (Mausbach et al., 2005) and acute stress-induced changes in hemostasis (von Känel et al., 2004), help further develop a model of emotional distress as one of the key factors for cardiovascular risk among dementia caregivers.

Identifying the existence of both depression and caregiver reaction to problem behaviors as predictors of time to cardiovascular disease provides a clearly definable and specific target for minimizing the potential negative health consequences of caregiving. For example, it appears possible that psychosocial interventions, which have been shown to effectively reduce depressive symptoms (Brodaty \& Hadzi-Pavlovic, 1990; Mittelman, Roth, Coon, \& Haley, 2004; Sörensen, Pinquart, \& Duberstein, 2002) and burden (Mittelman, Roth, Haley, \& Zarit, 2004; Sörensen et al., 2002) among dementia caregivers, may also serve to enhance caregiver health. Specifically, treatments that help caregivers better manage depressive symptoms and reaction to problem behaviors, including psychoeducational and multicomponent interventions (Depp et al., 2003; Mittelman, Roth, Coon et al., 2004; Pusey \& Richards, 2001; Sörensen et al., 2002), may have a protective effect on caregiver cardiovascular functioning. However, these assumptions are still preliminary and should be tested empirically. It is therefore recommended that future research examine whether a reduction in emotional distress via treatments can also confer a reduced risk for developing CVD over time.

These findings point to the importance of helping caregivers gain access to these interventions as a potential means of reducing not only the emotional distress of caregiving but also physical morbidity. Primary care physicians and other health service providers, often the principal source from whom caregivers seek help, appear to be in the best position to identify early signs of depression and distress among caregivers. Providers who use effective screening tools for early detection of emotional distress and who provide a means of receiving early interventions may help caregivers reduce their risk for both emotional distress and depression. This, in turn, may help reduce risk of developing cardiovascular disease.

It is important to note that additional clinical evaluations or reviews of caregivers' medical records were not conducted to confirm a diagnosis of cardiovascular disease. Rather, caregivers indicated whether a physician had diagnosed them with cardiovascular disease. While it is possible this may have affected our results, it is unlikely given that self-report accuracy for heart disease is very high (Bergmann, Byers, Freedman, \& Mokdad, 1998), with over $90 \%$ accuracy being consistently reported in previous research (Bush, Miller, Golden, \& Hale, 1989; Lee et al., 2003; Wolfe \& Michaud, 2004). Nonetheless, future studies should validate these findings using more strict assessments of cardiovascular disease diagnosis.

This study was limited to 18-months of follow-up, and the relative brevity of this study limited the number of participants who developed cardiovascular disease. Although both depression and reaction to problem behaviors emerged as significant predictors of time to CVD, the overall risk for developing CVD over our 18-month interval was low (i.e., 5\%). Therefore, we urge caution in interpreting the magnitude of the relationship between depression and distress on CVD risk and encourage replication of these findings.

Recent evidence suggests that depressive symptoms and/or distress may promote exaggerated catecholamine response to mental (Mausbach et al., 2005) and physical stress (Rudorfer, Ross, Linnoila, Sherer, \& Potter, 1985), which may lead to "downstream" effects such as platelet activity (Nemeroff \& Musselman, 2000) and elevations in the procoagulant factor D-dimer (von Känel \& Dimsdale, 2003). Perhaps a longer follow-up time period would have helped identify if depression and distress indeed predict long-term time to CVD onset rather than the more imminent onset of 18 months. In addition, future research should consider using depressive symptoms and reaction as timedependent covariates in order to understand, for example, the importance of depressive symptoms at varying time-points throughout the study can significantly predict time to onset.

Caregivers of dementia patients experience multiple stressors, and this study was limited to assessment of caregiver depressive symptoms and reaction to care recipient problem behaviors. Future studies should expand our research by including other measures of stress/distress. These may include measures of care recipient cognitive functioning and need for help with activities of daily living (e.g., Folstein, Folstein, \& McHugh, 1975; Hughes, Berg, Danziger, Coben, \& Martin, 1982; Katz, Ford, Moscowitz, Jackson, \& Jaffee, 1963), and assessments of caregiver role overload, family conflict, deprivation of intimate exchange, burden, mastery, coping, and social support (e.g., Bédard et al., 2001; Folkman \& Lazarus, 1985; Pearlin, Mullan, Semple, \& Skaff, 1990).

In sum, the current study provides preliminary evidence that depression and distress, conceptualized as reaction to care recipient problem behaviors, may shorten the time it takes caregivers to develop cardiovascular disease. Although this risk is relatively low during an 18-month follow-up period, the absence of early detection and prevention of depression and emotional distress may place caregivers at higher risk for early mortality. The present study 
opens doors for future exploration of caregiving factors that predict the onset of cardiovascular disease and to explore whether or not treatments that reduce emotional distress delay and/or prevent the onset of cardiovascular disease.

\section{References}

Anda, R., Williamson, D., Jones, D., Macera, C., Eaker, E., Glassman, A., et al. (1993). Depressed affect, hopelessness, and the risk of ischemic heart disease in a cohort of U.S. adults. Epidemiology, 4, 285-294.

Bédard, M., Molloy, D. W., Squire, L., Dubois, S., Lever, J. A., \& O'Donnell, M. (2001). The Zarit Burden Interview: A new short version and screening version. The Gerontologist, 41, 652-657.

Baumgarten, M., Battista, R. N., Infante-Rivard, C., Hanley, J. A., Becker, R., \& Gauthier, S. (1992). The psychological and physical health of family members caring for an elderly person with dementia. Journal of Clinical Epidemiology, 45, 61-70.

Bergmann, M. M., Byers, T., Freedman, D. S., \& Mokdad, A. (1998). Validity of self-reported diagnoses leading to hospitalization: A comparison of self-reports with hospital records in a prospective study of American adults. American Journal of Epidemiology, 147, 969-977.

Brodaty, H., \& Hadzi-Pavlovic, D. (1990). Psychosocial effects on carers of living with persons with dementia. The Australian and New Zealand Journal of Psychiatry, 24, 351-361.

Bush, T. L., Miller, S. R., Golden, A. L., \& Hale, W. E. (1989). Self-report and medical record report agreement of selected medical conditions in the elderly. American Journal of Public Health, 79, 1554-1556.

Cohen, D., Luchins, D., Eisdorfer, C., Paveza, G. J., Ashford, J. W., Gorelick, P., et al. (1990). Caring for relatives with Alzheimer's disease: The mental health risks to spouses, adult children, and other family caregivers. Behavior, Health, \& Aging, 1, 171-182.

Depp, C., Krisztal, E., Cardenas, V., Oportot, M., Mausbach, B., Ambler, C., et al. (2003). Treatment Options for Improving Wellbeing in Dementia Family Caregivers: The Case for Psychoeducational Interventions. Clinical Psychologist, 7, 21-31.

Folkman, S., \& Lazarus, R. S. (1985). If it changes it must be a process: Study of emotion and coping during three stages of a college examination. Journal of Personality and Social Psychology, 48, 150-170.

Folstein, M. F., Folstein, S. E., \& McHugh, P. R. (1975). "Mini-mental state". A practical method for grading the cognitive state of patients for the clinician. Journal of Psychiatric Research, 12, 189-198.

Ford, D. E., Mead, L. A., Chang, P. P., Cooper-Patrick, L., Wang, N. Y., \& Klag, M. J. (1998). Depression is a risk factor for coronary artery disease in men: The precursors study. Archives of Internal Medicine, $158,1422-1426$

Grant, I. (1999). Caregiving may be hazardous to your health. Psychosomatic Medicine, 61, 420-423.

Hughes, C. P., Berg, L., Danziger, W. L., Coben, L. A., \& Martin, R. L. (1982). A new clinical scale for the staging of dementia. The British Journal of Psychiatry; The Journal of Mental Science, 140, 566-572.

Katz, S., Ford, A. B., Moscowitz, R. W., Jackson, B. A., \& Jaffee, M. W. (1963). Studies of illness in the aged. The index of ADL: A standardized measure of biological and psychosocial function. Journal of the American Medical Association, 185, 914-919.

Kiecolt-Glaser, J. K., Glaser, R., Gravenstein, S., Malarkey, W. B., \& Sheridan, J. (1996). Chronic stress alters the immune response to influenza virus vaccine in older adults. Proceedings of the National Academy of Sciences of the United States of America, 93, 3043-3047.

Kiecolt-Glaser, J. K., Marucha, P. T., Malarkey, W. B., Mercado, A. M., \& Glaser, R. (1995). Slowing of wound healing by psychological stress. Lancet, 346, 1194-1196.

Lee, S., Colditz, G. A., Berkman, L. F., \& Kawachi, I. (2003). Caregiving and risk of coronary heart disease in U.S. women: A prospective study. American Journal of Preventive Medicine, 24, 113-119.
Lespérance, F., Frasure-Smith, N., Talajic, M., \& Bourassa, M. G. (2002). Five-year risk of cardiac mortality in relation to initial severity and one-year changes in depression symptoms after myocardial infarction. Circulation, 105, 1049-1053.

Lip, G. Y., \& Lowe, G. D. (1995). Fibrin D-dimer: A useful clinical marker of thrombogenesis? Clinical Science, 89, 205-214.

Mausbach, B. T., Dimsdale, J. E., Ziegler, M. G., Mills, P. J., Ancoli-Israel, S., Patterson, T. L., et al. (2005). Depressive symptoms predict norepinephrine response to a psychological stressor task in Alzheimer's caregivers. Psychosomatic Medicine, 67, 638-642.

Mills, P. J., Adler, K. A., Dimsdale, J. E., Perez, C. J., Ziegler, M. G., Ancoli-Israel, S., et al. (2004). Vulnerable caregivers of Alzheimer disease patients have a deficit in beta 2 -adrenergic receptor sensitivity and density. The American Journal of Geriatric Psychiatry, 12, 281286.

Mills, P. J., Yu, H., Ziegler, M. G., Patterson, T., \& Grant, I. (1999). Vulnerable caregivers of patients with Alzheimer's disease have a deficit in circulating CD62L- T lymphocytes. Psychosomatic Medicine, 61, $168-174$.

Mills, P. J., Ziegler, M. G., Patterson, T., Dimsdale, J. E., Hauger, R., Irwin, M., et al. (1997). Plasma catecholamine and lymphocyte beta 2 -adrenergic receptor alterations in elderly Alzheimer caregivers under stress. Psychosomatic medicine, 59, 251-256.

Mittelman, M. S., Roth, D. L., Coon, D. W., \& Haley, W. E. (2004). Sustained benefit of supportive intervention for depressive symptoms in caregivers of patients with Alzheimer's disease. The American Journal of Psychiatry, 161, 850-856.

Mittelman, M. S., Roth, D. L., Haley, W. E., \& Zarit, S. H. (2004). Effects of a caregiver intervention on negative caregiver appraisals of behavior problems in patients with Alzheimer's disease: Results of a randomized trial. The Journals of Gerontology Series B, Psychological Sciences and Social Sciences, 59, P27-34.

Musselman, D. L., Evans, D. L., \& Nemeroff, C. B. (1998). The relationship of depression to cardiovascular disease: Epidemiology, biology, and treatment. Archives of general Psychiatry, 55, 580-592.

Nemeroff, C. B., \& Musselman, D. L. (2000). Are platelets the link between depression and ischemic heart disease? American Heart Journal, 140, 57-62.

Pearlin, L. I., Mullan, J. T., Semple, S. J., \& Skaff, M. M. (1990). Caregiving and the stress process: An overview of concepts and their measures. The Gerontologist, 30, 583-594.

Pelzer, H., Schwarz, A., \& Heimburger, N. (1988). Determination of human thrombin-antithrombin III complex in plasma with an enzymelinked immunosorbent assay. Thrombosis and Haemostasis, 59, 101106.

Pike, J. L., Smith, T. L., Hauger, R. L., Nicassio, P. M., Patterson, T. L., McClintick, J., et al. (1997). Chronic life stress alters sympathetic, neuroendocrine, and immune responsivity to an acute psychological stressor in humans. Psychosomatic Medicine, 59, 447-457.

Pusey, H., \& Richards, D. (2001). A systematic review of the effectiveness of psychosocial interventions for carers of people with dementia. Aging \& Mental Health, 5, 107-119.

Radloff, L. S. (1976). The CES-D Scale: A self-report depression scale for research in the general population. Applied Psychological Measurement, $1,385-401$

Radloff, L. S., \& Teri, L. (1986). Use of the Center for Epidemiological Studies-Depression Scale with older adults. Clinical Gerontologist, 5, $119-136$.

Rudorfer, M. V., Ross, R. J., Linnoila, M., Sherer, M. A., \& Potter, W. Z. (1985). Exaggerated orthostatic responsivity of plasma norepinephrine in depression. Archives of General Psychiatry, 42, 1186-1192.

Sörensen, S., Pinquart, M., \& Duberstein, P. (2002). How effective are interventions with caregivers? An updated meta-analysis. The Gerontologist, 42, 356-372. 
Schulz, R., \& Beach, S. R. (1999). Caregiving as a risk factor for mortality: The Caregiver Health Effects Study. Journal of the American Medical Association, 282, 2215-2219.

Schulz, R., Burgio, L., Burns, R., Eisdorfer, C., Gallagher-Thompson, D., Gitlin, L. N., et al. (2003). Resources for Enhancing Alzheimer's Caregiver Health (REACH): overview, site-specific outcomes, and future directions. The Gerontologist, 43, 514-520.

Schulz, R., O’Brien, A. T., Bookwala, J., \& Fleissner, K. (1995). Psychiatric and physical morbidity effects of dementia caregiving: Prevalence, correlates, and causes. The Gerontologist, 35, 771-791.

Shaw, W. S., Patterson, T. L., Ziegler, M. G., Dimsdale, J. E., Semple, S. J., \& Grant, I. (1999). Accelerated risk of hypertensive blood pressure recordings among Alzheimer caregivers. Journal of Psychosomatic Research, 46, 215-227.

Teri, L., Truax, P., Logsdon, R., Uomoto, J., Zarit, S., \& Vitaliano, P. P. (1992). Assessment of behavioral problems in dementia: The revised memory and behavior problems checklist. Psychology and Aging, 7, 622-631.

Vitaliano, P. P., Russo, J., \& Niaura, R. (1995). Plasma lipids and their relationships with psychosocial factors in older adults. The Journals of Gerontology Series B, Psychological Sciences and Social Sciences, 50, P18-P24.

Vitaliano, P. P., Scanlan, J. M., Krenz, C., \& Fujimoto, W. (1996). Insulin and glucose: Relationships with hassles, anger, and hostility in nondiabetic older adults. Psychosomatic Medicine, 58, 489-499.

Vitaliano, P. P., Scanlan, J. M., Zhang, J., Savage, M. V., Hirsch, I. B., \& Siegler, I. C. (2002). A path model of chronic stress, the metabolic syndrome, and coronary heart disease. Psychosomatic Medicine, 64, $418-435$.

Vitaliano, P. P., Zhang, J., \& Scanlan, J. M. (2003). Is caregiving hazard- ous to one's physical health? A meta-analysis. Psychological Bulletin, 129, 946-972.

von Känel, R., \& Dimsdale, J. E. (2003). Fibrin D-dimer: A marker of psychosocial distress and its implications for research in stress-related coronary artery disease. Clinical Cardiology, 26, 164-168.

von Känel, R., Dimsdale, J. E., Adler, K. A., Patterson, T. L., Mills, P. J., \& Grant, I. (2004). Effects of depressive symptoms and anxiety on hemostatic responses to acute mental stress and recovery in the elderly. Psychiatry Research, 126, 253-264.

von Känel, R., Dimsdale, J. E., Ziegler, M. G., Mills, P. J., Patterson, T. L., Lee, S. K., et al. (2001). Effect of acute psychological stress on the hypercoagulable state in subjects (spousal caregivers of patients with Alzheimer's disease) with coronary or cerebrovascular disease and/or systemic hypertension. The American Journal of Cardiology, 87, 14051408.

von Känel, R., Dimsdale, J. E., Adler, K. A., Patterson, T. L., Mills, P. J., \& Grant, I. (2005). Exaggerated plasma fibrin formation (D-dimer) in elderly Alzheimer caregivers as compared to noncaregiving controls. Gerontology, 51, 7-13.

Ware, J., Jr., Kosinski, M., \& Keller, S. D. (1996). A 12-Item Short-Form Health Survey: Construction of scales and preliminary tests of reliability and validity. Medical Care, 34, 220-233.

Williamson, G. M., \& Schulz, R. (1993). Coping with specific stressors in Alzheimer's disease caregiving. The Gerontologist, 33, 747-755.

Wisniewski, S. R., Belle, S. H., Coon, D. W., Marcus, S. M., Ory, M. G., Burgio, L. D., et al. (2003). The Resources for Enhancing Alzheimer's Caregiver Health (REACH): project design and baseline characteristics. Psychology and Aging, 18, 375-384.

Wolfe, F., \& Michaud, K. (2004). Heart failure in rheumatoid arthritis: Rates, predictors, and the effect of anti-tumor necrosis factor therapy. The American Journal of Medicine, 116, 305-311. 